\title{
Patient and in vitro fertilization (IVF) cycle characteristics associated with variable blastulation rates: a retrospective study from the Duke Fertility Center (2013-2017)
}

\author{
Carrie A. Jones ${ }^{1,2^{*}}$, Kelly S. Acharya ${ }^{1}$, Chaitanya R. Acharya ${ }^{3}$, Douglas Raburn ${ }^{1}$ and Suheil J. Muasher ${ }^{1}$
}

\begin{abstract}
Background: To evaluate the association of patient and IVF cycle characteristics with blastulation rate and formation of high-quality blastocysts

Results: We analyzed autologous blastocyst cycles from 2013 to 2017. Cycles were subdivided into low (<33\%), intermediate (33-66\%), and high (>66\%) blastulation rates. Embryo quality was assigned by embryologists using Gardner Criteria. R statistical package was used, and the blastulation groups were compared using analysis of variance (ANOVA) for continuous variables and chi-squared tests for categorical variables. The Bonferroni correction was used to adjust for multiple comparisons. One hundred seventeen IVF cycles met our inclusion criteria. Of these, 20 (17.1\%) had low, 74 (63.2\%) had intermediate, and 23 (19.7\%) had high blastulation rates. Low blastulation rate was associated with a lower number of blastocysts, including fewer high-quality blastocysts. The mean number of oocytes retrieved was highest (18.1) in the group with the lowest blastulation rate, and lowest (13.4) in those with the highest blastulation rate, although this did not reach statistical significance. There were no significant differences between blastulation rates and age, gravidity, prior live birth, anti-mullerian hormone, estradiol and progesterone levels on the day of ovulation trigger, follicle-stimulating hormone dose, or fertility diagnosis.

Conclusions: High blastulation rate is associated with a greater number of blastocysts, including a greater number of high-quality blastocysts. Higher oocyte yield, however, is not associated with improved blastulation rates. Blastulation rates, blastocyst number, and quality remain difficult to predict based on cycle characteristics alone, and oocyte yield may not be an accurate predictor of either outcome.
\end{abstract}

\section{Precis}

High oocyte yield may be associated with a lower blastulation rate and a lower number of high-quality blastocysts.

\section{Background}

The transfer of blastocysts during in vitro fertilization (IVF) cycles offers several advantages to the transfer of cleavage stage embryos. These include, but are not limited to, increased implantation rates, increased live birth rates, improved selection of embryos based on

\footnotetext{
* Correspondence: Carrie.Jones@duke.edu

${ }^{1}$ Division of Reproductive Endocrinology and Infertility, Duke University

Medical Center, 5704 Fayetteville Road, Durham, NC 27713, USA

25324 Mcfarland Rd Ste 300, Durham, NC 27707, USA

Full list of author information is available at the end of the article
}

morphology, better synchronization of endometrium and embryo at the time of transfer, and ability to perform preimplantation genetic testing for aneuploidy (PGT-A) [1]. Transfer of blastocysts of the highest morphologic quality is also associated with improved clinical pregnancy rates $[2,3]$. The improved IVF outcomes associated with blastocyst embryo transfer allow for the transfer of only one embryo for most patients, which is associated with a lower rate of multiple gestations $[4,5]$.

At each step of an IVF cycle, there is a potential for attrition of the number of possible viable embryos. Not all retrieved oocytes will be mature, and not all oocytes will become fertilized embryos. It is difficult to predict which cleavage stage embryos (days $2-3$ ), if any, will become viable blastocysts (days 5-6). It is also impossible 
to know which of those cleavage stage embryos, if any, will become high-quality blastocysts. The ability to better predict the expected number and quality of blastocysts during an IVF cycle would have a significant impact on the way that those patients are counselled and potentially on how their IVF cycles are managed.

Certain IVF cycle and patient characteristics may be associated with improved blastulation rates and increased formation of high-quality blastocysts. Previous studies have shown that lower blastulation rates are associated with aneuploid embryos [6]. Superovulation in mouse embryos has been shown to decrease blastulation rates $[7,8]$. High progesterone level at the time of trigger has been associated with decreased formation of top-quality blastocysts [9]. High blastulation rates and formation of highquality embryos is a clinically important outcome, and currently, there is a paucity of data to guide clinical practice towards improving these outcomes.

The main objective of this study was to evaluate the patient and IVF cycle characteristics resulting in high blastulation rates and that result in the formation of the highest quality embryos.

\section{Methods}

This is a retrospective study of IVF cycles in patients at the Duke Fertility Center (DFC) from January 2013March 2017. The cohort of cycles included all cycles in which IVF patients completed an autologous IVF cycle and in which all embryos underwent extended culture in an attempt to reach the blastocyst stage (day 5-6); after excluding those cycles that did not meet the criteria, the cohort included 117 patient cycles. These patients, per the clinic's criteria, had at least five embryos on day 3 , each with seven cells or more and $<20 \%$ fragmentation. We included cycles for patients who had both conventional IVF and intracytoplasmic sperm injection (ICSI) as their mode of fertilization. We also included patients who underwent preimplantation genetic testing for aneuploidy (PGT-A). We excluded all oocyte donor cycles and those cycles in which sperm was retrieved surgically. We also excluded any cycles that involved the cryopreservation or transfer of any cleavage-stage embryos. The study was approved by the Duke Health Institutional Review Board.

We retrospectively reviewed records of all IVF cycles meeting the above inclusion criteria during the study period. Demographic data were abstracted from patient charts including age, height, weight, ethnicity, gravidity, and prior live births. Body mass index (BMI) was calculated as weight $(\mathrm{kg}) /[$ height $(\mathrm{m})]$ [2]. We also collected data on other baseline patient characteristics including ovarian reserve testing (anti-mullerian hormone, $\mathrm{AMH}$, levels) and infertility diagnosis. Of note, if multiple infertility diagnoses were listed for a patient, both diagnoses were included for that patient's cycle, and they were both used in the calculations for the impact of infertility diagnosis on blastulation cohort. IVF cycle characteristics including fertilization method, estradiol (E2) and progesterone (P4) levels on the day of ovulation trigger, and total follicle-stimulating hormone (FSH) dose were also obtained. IVF cycle outcomes were collected, including the number of oocytes retrieved, the number of fertilized oocytes, the total number of blastocysts formed, and the number of high-quality blastocysts.

Blastulation rate was defined as the total number of blastocysts in a cycle (including those transferred and cryopreserved) divided by the total number of fertilized oocytes. The percent of high-quality blastocysts was defined as the number of high-quality blastocysts divided by the number of total fertilized oocytes in a particular cycle. Blastulation rates were subdivided into low $(<33 \%)$, intermediate (33-66\%), and high (>66\%) for analysis.

The quality of each embryo at day 5-6 was reviewed and recorded by trained embryologists at the DFC using the Gardner Criteria. Using these criteria, the expansion or hatching grade, inner cell mass (ICM), and trophectoderm (TE) quality of each embryo was assigned a standard alphanumeric rating. High-quality blastocyst was defined as grade 3 development or better, ICM grade A or $\mathrm{B}$, and TE grade A or B, with either ICM or TE, or both, being graded as A.

To reach a grade 3 (or higher) in embryo expansion, the embryo must be at least $50 \%$ blastocoel with either a very thin zona pellucida or with noted herniation through the zona, with hatching either partial or full. For ICM to obtain a grade A, the ICM must be noted to be compact and tightly packed, with a grade $\mathrm{B}$ given to ICM showing loose, larger cells that are less densely packed. TE to receive a grade A must show many cohesive cells, whereas a grade B includes scant but large cells within the TE. High-quality blastocysts were similarly defined in the Vanni study [9].

$\mathrm{R}$ statistical package was used, and the blastulation groups were compared using analysis of variance (ANOVA) for continuous variables and chi-squared tests for categorical variables. The Bonferroni correction was used to adjust for multiple comparisons. Statistical significance with the Bonferroni correction was set at 0.0025 .

\section{Results}

A total of 117 patient cycles were identified that met the inclusion criteria during the study period. Mean patient age was 32.7 years. The majority of patients were white (Table 1). Average blastulation rate for all cycles was $49.5 \%$, with an average of 5 blastocysts per cycle and an average of 2.5 high-quality blastocysts per cycle. The 
Table 1 Overall patient and cycle characteristics

\begin{tabular}{ll}
\hline Characteristic & 117 \\
\hline Total cycles & $32.7 \pm 3.7$ \\
Age (years) & \\
Race & $82(70.1)$ \\
$\quad$ White & $3(2.6)$ \\
Black or African American & $14(12.0)$ \\
Asian & $16(13.7)$ \\
Other & $2(1.7)$ \\
Unknown & $16.7 \pm 7.6$ \\
Number of oocytes retrieved & $10.4 \pm 5.0$ \\
Number of fertilized oocytes & 49.5 \\
Blastulation rate overall (\%) & $5 \pm 2.9$ \\
Number of blastocysts & $2.3 \pm 2.1$ \\
Number of high-quality blastocysts
\end{tabular}

Data are mean \pm SD or $n(\%)$ unless otherwise specified

mean number of oocytes retrieved for all cycles was 16.7.

The mean patient age was 33.4 years in the low blastulation rate group versus 32.1 years in the high blastulation rate group (Table 2, $p=0.508$ ). Patients with the highest rate of blastulation had a mean gravidity of 1.0, while patients with the lowest rates of blastulation had a mean gravidity of $0.6(p=0.462) .30 .4 \%$ of patients in the high blastulation cohort had a prior live birth, compared to $15.0 \%$ in those with low blastulation rate $(p=0.488)$.

There was no difference in blastulation rates by fertility diagnosis (Table 3). The highest percentage of patients in the low blastulation group was undergoing preimplantation genetic testing (PGT-A). The most common reason for infertility in the intermediate and high blastulation rate groups was male factor infertility (32.4 and $43.5 \%$, respectively).

The vast majority patients in all groups underwent ICSI for fertilization method rather than conventional IVF (Table 4). E2 and P4 levels were not statistically significant between groups (Table 4). Similarly, FSH dose was not statistically significant between groups $(p=0.100)$

The mean number of oocytes retrieved was 18.1 in the patients with low blastulation versus 13.4 in those with high blastulation (Table 5, $p=0.060$ ). A higher number of fertilized oocytes was seen in those with low blastulation rates $(p=0.021)$. Those with high blastulation rates had a significantly higher number of blastocysts (6.00) compared to the low blastulation rate group (2.45). Those with the highest blastulation rates also had a significantly higher average number and percent of highquality blastocysts $(p=0.0002$ and $<0.0001)$. Although examination of clinical outcomes (beyond blastulation) was not the primary objective of the study, the clinical pregnancy rate was examined in the cycles in which fresh transfer was attempted (Table 4). No association was found between the blastulation rate and the clinical pregnancy rate $(p=0.90)$.

\section{Discussion}

The results of our study suggest that patients with high rates of blastulation are, as may be expected, inherently more likely to yield more high-quality blastocysts during IVF. These patients with high blastulation rates are not necessarily the patients with the most oocytes retrieved, which may surprise some patients who believe that high oocyte yield is associated with universally better outcomes. Improved blastulation rate and formation of

Table 2 Patient characteristics by variable blastulation rates

\begin{tabular}{|c|c|c|c|c|}
\hline & $\begin{array}{l}\text { Low blastulation } \\
(<33 \%)\end{array}$ & $\begin{array}{l}\text { Intermediate blastulation } \\
(33-66 \%)\end{array}$ & $\begin{array}{l}\text { High blastulation } \\
(>66 \%)\end{array}$ & $p$ \\
\hline$n$ & 20 & 74 & 23 & $\mathrm{n} / \mathrm{a}$ \\
\hline$\%$ in cohort & 17.1 & 63.2 & 19.7 & $\mathrm{n} / \mathrm{a}$ \\
\hline Age (years) & $33.4 \pm 4.9$ & $32.7 \pm 3.5$ & $32.1 \pm 3.0$ & 0.508 \\
\hline \multicolumn{5}{|l|}{ Race } \\
\hline White & $14(73.7)$ & $51(69.9)$ & 17 (73.9) & 0.903 \\
\hline Black & $0(0.0)$ & $3(4.1)$ & $0(0.0)$ & 0.412 \\
\hline Asian & $3(15.8)$ & $8(11.0)$ & $3(13.0)$ & 0.839 \\
\hline Other & $2(10.5)$ & $11(15.1)$ & $3(13.0)$ & 0.870 \\
\hline BMI $\left(\mathrm{kg} / \mathrm{m}^{2}\right)$ & $25.2 \pm 4.3$ & $23.9 \pm 3.3$ & $24.8 \pm 5.3$ & 0.405 \\
\hline AMH (ng/mL) & $5.02 \pm 5.3$ & $5.56 \pm 5.8$ & $4.56 \pm 2.7$ & 0.705 \\
\hline Gravidity & $0.60 \pm 0.8$ & $0.82 \pm 1.1$ & $1.0 \pm 1.4$ & 0.462 \\
\hline Prior live birth & $3(15.0)$ & $17(23.0)$ & $7(30.4)$ & 0.488 \\
\hline
\end{tabular}

Data are mean + SD or $n$ (\%) unless otherwise specified $B M I$ body mass index, $A M H$ anti-mullerian hormone 
Table $\mathbf{3}$ Infertility diagnosis for variable blastulation rates

\begin{tabular}{|c|c|c|c|c|}
\hline & $\begin{array}{l}\text { Low blastulation } \\
(<33 \%)\end{array}$ & $\begin{array}{l}\text { Intermediate blastulation } \\
(33-66 \%)\end{array}$ & $\begin{array}{l}\text { High blastulation } \\
(>66 \%)\end{array}$ & $p$ \\
\hline Male factor & $11(5.5)$ & $24(32.4)$ & $10(43.5)$ & 0.158 \\
\hline Tubal factor & $2(10)$ & $7(9.5)$ & $1(4.3)$ & 0.722 \\
\hline PCOS & $1(5.0)$ & $11(14.9)$ & $4(17.4)$ & 0.442 \\
\hline Unexplained & $3(15.0)$ & $23(31.1)$ & $4(17.4)$ & 0.206 \\
\hline PGT-A & $5(25.0)$ & 14 (18.9) & $9(39.1)$ & 0.139 \\
\hline RPL & $2(10)$ & $5(6.6)$ & $3(13.0)$ & 0.621 \\
\hline
\end{tabular}

Data are mean + SD or $n(\%)$ unless otherwise specified

$P C O S$ polycystic ovarian syndrome, PGT-A preimplantation genetic testing for aneuploidy, RPL recurrent pregnancy loss ( $\geq 2$ spontaneous abortions)

high-quality embryos were not found to be associated with any particular patient or IVF cycle characteristics. This finding highlights the fact that IVF cycle outcomes, including blastulation rate and formation of high-quality embryos, are difficult to predict.

It is unknown whether infertility diagnosis may impact blastulation rates or likelihood of formation of topquality embryos. Infertility diagnoses have in prior studies been correlated with variable obstetric outcomes after IVF. Specifically, ovulatory dysfunction and PCOS have been associated with poorer obstetric outcomes [10]. In a large retrospective cohort study, higher blastulation rate was associated with a higher incidence of tubal and uterine factor infertility, whereas low blastulation rates were associated with a higher rate of unexplained infertility [11]. The potential for variable IVF outcomes by infertility diagnosis is an area for further research. Of note, the percentage of cycles in which ICSI was performed was significantly higher than the percentage of cycles in which male factor infertility was listed as the diagnosis (Tables 2 and 3). The decision to perform ICSI in the DFC is made on a provider-by-provider basis, and ICSI is performed regularly for certain other clinical scenarios such as planned PGT-A, unexplained infertility (with the thought that fertilization defect may be the yet-unknown cause of infertility for a certain couple), frozen sperm, and nulliparous patients who have never had proven fertilization potential with their partner's sperm.

IVF outcomes have been shown to be impacted by patient ethnicity, BMI, and age. Our study did not show any such association, but it is possible that the sample size was too small to detect any difference. We would expect worse blastulation rates and lower formation of top-quality embryos in patients with high BMI and who were older. In one large retrospective cohort analysis, blastulation rates were significantly lower in older patients [11]. The oldest patients in our study did have the lowest blastulation rates, but these results did not reach statistical significance.

E2 and P4 levels on the day of ovulation trigger are routinely tracked in IVF cycles. Elevated P4 is thought to have detrimental effects on endometrial receptivity, and cycles with elevated progesterone are typically managed with a freeze-all technique rather than with fresh embryo transfer [9]. There is some evidence to suggest that elevated progesterone also results in a decreased formation of highquality embryos [9]. Progesterone has been found to be an independent predictor of pregnancy in IVF cycles, with higher P4 levels being associated with a decreased likelihood of pregnancy [10]. While higher progesterone was noted in patients with lower blastulation rates in our study, this did not reach statistical significance.

There are no prior studies to our knowledge that assess E2 levels and their association with formation of top-quality embryos. Our study did not show any such association, but it may be that there were an inadequate number of available subjects to detect any difference. Blastulation rates and blastocyst quality associated with E2 and P4 levels are an area for further investigation.

The finding of a lower number of retrieved oocytes in those patients with the higher blastulation rates (although

Table 4 IVF cycle characteristics for variable blastulation rates

\begin{tabular}{lllll}
\hline & $\begin{array}{l}\text { Low blastulation } \\
(<33 \%)\end{array}$ & $\begin{array}{l}\text { Intermediate blastulation } \\
(33-66 \%)\end{array}$ & $\begin{array}{l}\text { High blastulation } \\
(>66 \%)\end{array}$ \\
\hline ICSI & $19(95.0)$ & $61(82.4)$ & $22(95.7)$ & $p$ \\
E2 $(\mathrm{pg} / \mathrm{mL})$ & $2548 \pm 932$ & $2667 \pm 1140$ & $2249 \pm 503$ & 0.131 \\
P4 $(\mathrm{ng} / \mathrm{mL})$ & $1.24 \pm 0.31$ & $1.21 \pm 0.42$ & $1.14 \pm 0.39$ & 0.229 \\
FSH dose $(\mathrm{IU})$ & $2255 \pm 1097$ & $2190 \pm 934$ & $2701 \pm 1004$ & 0.639 \\
\hline
\end{tabular}

Data are mean + SD or $n(\%)$ unless otherwise specified

ICSI intracytoplasmic sperm injection, E2 estrogen, $P 4$ progesterone, FSH follicle-stimulating hormone 
Table 5 IVF cycle outcomes by variable blastulation rates

\begin{tabular}{|c|c|c|c|c|}
\hline & $\begin{array}{l}\text { Low blastulation } \\
(<33 \%)\end{array}$ & $\begin{array}{l}\text { Intermediate blastulation } \\
(33-66 \%)\end{array}$ & $\begin{array}{l}\text { High blastulation } \\
(>66 \%)\end{array}$ & $p$ \\
\hline Oocytes retrieved & $18.05 \pm 7.9$ & $17.35 \pm 8.2$ & $13.35 \pm 4.2$ & 0.0600 \\
\hline Oocytes fertilized* & $11.50 \pm 6.14$ & $10.96 \pm 4.87$ & $7.91 \pm 3.4$ & 0.021 \\
\hline Blastulation rate (\%) & 22.0 & 48.6 & 76.5 & $<0.0001$ \\
\hline Number of blastocysts & $2.45 \pm 1.3$ & $5.39 \pm 2.9$ & $6.00 \pm 2.7$ & $<0.0001$ \\
\hline Number of $\mathrm{HQB}^{* *}$ & $0.65 \pm 0.7$ & $2.47 \pm 2.2$ & $3.09 \pm 2.1$ & 0.0002 \\
\hline $\mathrm{HQB}^{* * *}(\%)$ & 5.01 & 21.7 & 39.4 & $<0.0001$ \\
\hline$C P R^{* * * *}(\%)$ & 53.8 & 46.7 & 50.0 & 0.90 \\
\hline
\end{tabular}

Data are mean + SD or $n$ (\%) unless otherwise specified

*2PN or 2 pronūclei (represents the number of viable day 2 embryos)

${ }^{*} \mathrm{HQB}$, high-quality blastocysts

***The percentage of blastocysts that were deemed high-quality blastocysts by Gardner Criteria

****CPR, clinical pregnancy rate when fresh transfer attempted

this did not reach statistical significance) is an intriguing finding that warrants additional discussion. This finding was also reported by our group in a large retrospective analysis of 70,968 cycles, where those with the highest oocyte yield had the lowest blastulation rates [11]. Oocytes are, of course, essential in the formation of blastocysts for successful IVF cycles, but it is increasingly being recognized that a greater number of oocytes retrieved may not necessarily be associated with the best IVF outcomes [12, $14-18,21]$. It has been suggested that the optimal number of oocytes retrieved in a cycle may depend on the exact stimulation protocol and that a lower number of oocytes retrieved is not necessarily correlated with poorer IVF outcomes [12, 15, 17, 22]. This data suggests that an emphasis on embryo quality over oocyte quantity may be a more appropriate strategy for the management of IVF cycles. Despite this evidence, low oocyte retrieval remains a feared outcome for patients and providers alike.

More "mild" ovarian stimulation during IVF cycles has several benefits, including decreased incidence of ovarian hyperstimulation syndrome (OHSS), decreased frequency of multiple gestations, decreased rates of aneuploidy, lower cost, and improved access to IVF treatment [19]. Milder stimulation protocols have been associated with better implantation and pregnancy rates despite a lower number of oocytes retrieved [19]. Multiple mechanisms to explain this paradox that less oocytes may yield improved IVF outcomes have been proposed. Elevated E2 and P4 levels seen with conventional IVF protocols may be associated with direct embryo toxicity and diminished endometrial receptivity, and as such have poorer IVF outcomes [19]. It has been suggested that elevated E2 may disrupt folliculogenesis and oocyte maturation. Incidence of low birth weight, preeclampsia, and longterm health consequences for IVF offspring have been shown to be increased in patients with supraphysiologic E2 concentrations in early gestation [19]. Therefore, excessive E2 levels noted in aggressive stimulation protocols are best avoided. Available evidence supports that gentler ovarian stimulation selects for the best quality follicles and creates better quality oocytes and embryos [19]. However, it is important to note that all of the patients in our study underwent conventional controlled ovarian hyperstimulation, and the mean number of oocytes retrieved in each cohort was between 13 and 18; these oocyte yields would be difficult to achieve with minimal stimulation protocols. We also acknowledge that there were no differences in the FSH dose per blastulation cohort, indicating that high FSH dose itself may not be the culprit affecting blastulation rates. As stated above, we hypothesize that the estradiol and/or progesterone levels reached by the patient (of which high responders are at particular risk of achieving high hormonal milieu by the time of trigger) may have more of an effect on the development of embryos than the stimulation protocol. Thus, we hypothesize that if, in a minimal stimulation cycle, a patient were to achieve an adequate oocyte yield to recommend extended culture of embryos, those embryos may likely display high rates of blastulation given the more physiologic hormonal milieu at the time of oocyte maturation trigger.

Blastocyst quality is highly pertinent clinically in choosing the most ideal embryos for transfer, which are associated with significantly improved clinical pregnancy and live birth rates in IVF $[2,3,9,17]$. Despite this, there is little data to guide reproductive endocrinologists on how to improve or predict blastulation rates and anticipated blastocyst quality. Improvement of blastulation rates and formation of high-quality embryos is an area for much future investigation in IVF research.

The strengths of this study include the availability of hormone levels and embryo quality for evaluation in our data set. This is the first study to our knowledge that investigates the association between estrogen levels, rates of blastulation, and embryo quality. Limitations of this 
study are inherent in its retrospective design, small sample size, and single site analysis. Also, the included cycles in this study are not representative of the overall population undergoing IVF, given that only those cycles with a good number and quality of cleavage stage embryos underwent extended culture to blastocysts.

\section{Conclusion}

In conclusion, high blastulation rates are associated with improved formation of high-quality blastocysts. Higher oocyte yield, however, are not associated with improved blastulation rates. Blastulation rates and blastocyst number and quality remain difficult to predict based on patient and cycle characteristics alone, and oocyte yield may not be an accurate predictor of these outcomes.

\begin{abstract}
Abbreviations
AMH: Anti-mullerian hormone; ANOVA: Analysis of variance; BMl: Body mass index; DFC: Duke Fertility Center; E2: Estradiol; FSH: Follicle-stimulating hormone; ICM: Inner cell mass; ICSI: Intracytoplasmic sperm injection; IRB: Institutional Review Board; IVF: In vitro fertilization; OHSS: Ovarian hyperstimulation syndrome; P4: Progesterone; PCOS: Polycystic ovarian syndrome; PGT-A: Preimplantation genetic testing for aneuploidy; TE: Trophectoderm
\end{abstract}

\section{Acknowledgements}

We acknowledge the Duke Fertility Clinic staff and patients for their assistance in this project.

\section{Authors' contributions}

KA conceived of the study. CJ and KA performed the data collection with assistance from DR. SA assisted with the abstract development and editing of the manuscript. KA and CA performed the statistical analysis. The authors have read and approved the final manuscript.

\section{Funding}

No funding was obtained for this study.

\section{Availability of data and materials}

Data was reviewed by authors in a secure electronic medical record.

\section{Ethics approval and consent to participate}

IRB approval was obtained for this study and no consent was required for participation of this retrospective study.

\section{Consent for publication}

Not indicated.

\section{Competing interests}

The authors declare that they have no competing interests.

\section{Author details}

${ }^{1}$ Division of Reproductive Endocrinology and Infertility, Duke University Medical Center, 5704 Fayetteville Road, Durham, NC 27713, USA. ${ }^{2} 5324$ Mcfarland Rd Ste 300, Durham, NC 27707, USA. ${ }^{3}$ Department of Surgery, Duke University Medical Center, 2323 Erwin Road, Durham, NC 27705, USA.

Received: 13 August 2019 Accepted: 6 September 2019 Published online: 16 October 2019

\section{References}

1. American Society for Reproductive Medicine (2013) Blastocyst culture and transfer in clinical-assisted reproduction: a committee opinion. Fertil Steril 99(3):667-672

2. Gardner et al (2000) Blastocyst score affects implantation and pregnancy outcome: towards a single blastocyst transfer. Fertil Steril 73(6):1155-1158
3. Goto et al (2011) Prediction of pregnancy rate by blastocyst morphological score and age based on 1,488 single frozen-thawed blastocyst transfer cycles. Fertil Steril 95(3):948-952

4. American Society for Reproductive Medicine (2017) Guidelines on the limits to the number of embryos to transfer: a committee opinion. Fertil Steril 107: 901-903

5. Marsh CA, Farr SL, Chang J, Kissin DM, Grainger DA, Posner SF, Macaluso M, Jamieson DJ (2012) Trends and factors associated with the day 5 embryo transfer, assisted reproductive technology surveillance, USA, 2001-2009. Hum Reprod 27:2325-2331

6. Vega M, Breborowicz A, Moshier EL, McGovern PG, Keltz MD (2014) Blastulation rates decline in a linear fashion from euploid to aneuploid embryos with single versus multiple chromosomal errors. Fertil Steril 102(2): 394-398

7. Beaumont J, Smith A (1975) Embryonic mortality during the pre- and postimplantation periods of pregnancy in mature mice after superovulation. J Reprod Fertil 45(3):437-438

8. Ertzeid G, Storeng R (1992) Adverse effects of gonadotrophin treatment on pre- and postimplantation development in mice. J Reprod Fertil 96(2):649-655

9. Vanni VS, Somigliana E, Reschini M, Pagliardini L, Marotta E et al (2017) Topquality blastocyst formation rates in relation to progesterone levels on the day of oocyte maturation in GnRH antagonist IVF/ICSI cycles. PLoS One 12(5):1-10

10. Cai Q, Wan F, Appleby D, Hu L, Zhang H (2014) Quality of embryos transferred and progesterone levels are the most important predictors of live birth after fresh embryo transfer: a retrospective cohort study. J Assist Reprod Genet 31(2):185-194

11. Acharya KA, Jones CA, Keyhan S, Raburn D, Acharya CR, Muasher SJ (2017) How do patient and in vitro fertilization (IVF) cycle characteristics impact blastulation rates? An analysis of 70,968 blastocyst cycles from the SART registry. Fertil Steril 108(3):e92

12. McAvey B et al (2011) How many eggs are needed to produce an assisted reproductive technology baby: is more always better? Fertil Steril 96(2):332-335

13. Grigorescu V, Zhang Y, Kissin DM, Sauber-Schatz E, Sunderam M, Kirby RS, Diop H, McKane P, Jamieson DJ (2014) Maternal characteristics and pregnancy outcomes after assisted reproductive technology by infertility diagnosis: ovulatory dysfunction versus tubal obstruction. Fertil Steril 101: 1019-1025

14. Inge G et al (2005) Oocyte number per live birth in IVF: were Steptoe and Edwards less wasteful? Hum Reprod 20:588-592

15. Verberg M, Eijkemans M, Macklon N et al (2009) The clinical significance of the retrieval of a low number of oocytes following mild ovarian stimulation for IVF: a meta-analysis. Hum Reprod Update 15(1):5-12

16. Hsu M, Wang C, Chen C, Tzeng C (2016) Impact of the number of retrieved oocytes on pregnancy outcome in in vitro fertilization. Taiwan J Obstet Gynecol 55(6):821-825

17. Niinimaki M, Veleva Z, Martikainen $H$ (2015) Embryo quality is the main factor affecting cumulative live birth rate after elective single embryo transfer in fresh stimulation cycles. Eur J Obstet Gynecol Reprod Biol 194: $131-135$

18. Lin Y, Chang S, Lan K, Huang H, Chang C, Tsai M, Kung F, Huang F (2003) Human oocyte maturity in vivo determine the outcome of blastocyst development in vitro. J Assist Reprod Genet 20(12):506-512

19. Blumenfield Z (2015) Why more is less and less is more when it comes to ovarian stimulation. J Assist Reprod Genet 32(12):1713-1719

20. Baart EB, Martini E, Eijkemans $\mathrm{mJ}$, Van Opstal D, Beckers NG, Verhoeff A et al (2007) Milder ovarian stimulation for in-vitro fertilization reduces aneuploidy in the human preimplantation embryo: a randomized controlled trial. Hum Reprod 22:980-988

21. Baart EB, Macklon NS, Fauser BJ (2009) Ovarian stimulation and embryo quality. Reprod BioMed Online 18(2):45-50

22. Sunkara SK, Rittenberg V, Raine-Fenning N, Bhattacharya S, Zamora J, Coomarasamy A (2011) Association between the number of eggs and live birth in IVF treatment: an analysis of 400,135 treatment cycles. Hum Reprod 26:1768-1774

\section{Publisher's Note}

Springer Nature remains neutral with regard to jurisdictional claims in published maps and institutional affiliations. 